\title{
Effects of Denervation on the Mid-term Results of Knee Joint Functions after Non-resurfaced Total Knee Arthroplasty
}

\author{
Ningning Liu ${ }^{1}$, Huanhuan $\mathrm{Su}^{2}$, Yuanhe Wang ${ }^{3}$, Yu Zhang ${ }^{1}$, Hongjian $\mathrm{Yu}^{1 *}$ \\ ${ }^{1}$ Department of Joint Surgery, Binzhou People's Hospital, Binzhou 256000, Shandong Province, China \\ ${ }^{2}$ Department of Gastroenterology, Binzhou People's Hospital, Binzhou 256000, Shandong Province, China \\ ${ }^{3}$ Department of Joint Surgery, Affiliated Hospital of Qingdao University, Qingdao 266000, Shandong Province, China
}

\begin{abstract}
Objective: Total knee arthroplasty (TKA) has become an effective treatment modality for end-stage osteoarthritis and rheumatoid arthritis. Postoperative problems such as anterior knee pain affect postoperative patient satisfaction. Patellar denervation has been proposed as a technique to relieve pain, but its efficacy remains controversial. This study evaluated the mid- and long-term effects of patellar denervation on postoperative knee joint functions, hoping to provide better guidance for clinical practice. Methods: This study is a prospective randomized controlled double-blind study. 58 patients undergoing bilateral non-resurfaced total knee arthroplasty were included and randomized into two groups. Both groups underwent total knee arthroplasty while patellar denervation was performed only on the experimental group. Information regarding whether if patellar denervation was performed were withheld from all patients and outcome assessors. All surgeries were performed by the same high-level professional physician, and the post-stable knee prosthesis system (PS Scorpio NRG PS, Stryker) was used during the surgeries. The knee joint functions were evaluated by professional assessors before and after surgery. The evaluation indicators mainly include KSS scoring, Western Ontario and McMaster Universities (WOMAC) scoring and Visual Analogue Scale (VAS), FJS scoring, etc. The follow-up period was 3 years and 5 years after surgery. Results: The experimental group had better KSS and FJS scores than the control group, the difference was statistically significant. There was no significant inter-group difference in WOMAC and VAS scores. Conclusion: The patellar denervation in TKA patients has positive effects on the mid- and long-term recovery of knee joint functions, and the postoperative satisfaction is better.
\end{abstract}

Key words: Patella denervation; Total knee arthroplasty; Anterior knee pain; Electrocautery; Functional recovery

Publication date: May, 2021; Publication online: 31 May, 2021

*Corresponding author: Hongjian Yu, yuhongjian2616@126.com

\section{Introduction}

With the increasing maturity of Total Knee Arthroplasty (TKA) technology and the continuous improvement in prosthesis design, the amount of TKA surgery is increasing, but postoperative satisfaction is still not ideal, and postoperative problems such as anterior knee pain affect patients satisfaction after surgery. According to statistics, the incidence of knee pain after the first TKA is $20 \%-40 \% \%^{[1,2]}$. Anterior knee pain after TKA is often the main cause of postoperative dissatisfaction, and it also affects the surgical effects and postoperative recovery progress ${ }^{[3]}$.
In view of the multifactorial nature of the mechanism of post-TKA anterior knee pain, we cannot confirm it only by a single-factor study. Currently, there are increasing researches on reducing post-TKA anterior knee pain, but there is no consensus on its etiology and treatment. Among them, the research on patella treatment has always been a hot topic, such as intraoperative patella replacement, patella shaping, improvement of patellofemoral trajectory, and patella denervation treatment $e t c^{[4-8]}$. Studies have found that intraoperative patella shaping does not improve the postoperative function of TKA and reduce the rate of 
long-term revision ${ }^{[9]}$. Patella denervation is one of the most widely used surgical procedures at present, and it is widely used in China and abroad, but its efficacy in improving TKA postoperative functions and reducing postoperative anterior knee pain remain controversial.

Patella denervation surgery was first proposed by the American scholar Vegaand it was gradually promoted and applied in clinical practice ${ }^{[9]}$. Van Jonbergen found that in some European countries, about $56 \%$ of surgeons administer patella denervation treatment during TKA without patella replacement, and about $32 \%$ administer denervation treatment during TKA with patella replacement ${ }^{[10]}$. The occurrence of anterior knee pain may be related to the nociceptive afferent fibers of substance $\mathrm{P}$ in the surrounding tissues $^{[11]}$. Denervation of the patella, that is, the loss of functions of all these receptors through cauterization and diathermy, theoretically may lead to local denervation of the patella, reduce postoperative knee pain, and improve postoperative knee functions ${ }^{[9]}$.

Although patella denervation is widely used in clinical practice, its effectiveness is still controversial. Domestic and foreign studies on the effects of patella denervation surgery on postoperative functions have also came to different conclusions $^{[12]}$. However, there are few randomized controlled studies through self-comparison and long-term follow-up. This study intended to study the effects of TKA patella denervation on postoperative knee joint functions and its true efficacy.

\section{Materials and methods}

Experimental design: prospective, randomized control, double-blind trial.

In strict accordance with the inclusion and exclusion criteria, 58 hospitalized patients who planned to undergo bilateral TKA during the same period were selected and divided into experimental group and control group by random number table scheme, and the double-blindness was maintained, that is, the surgeons, patients and assessors were all not aware of the grouping status. Knee joint function scores before and after surgery were completed by assessors who are familiar with knee joint physical examination and scoring. This mid-term clinical study was followed up for 3 and 5 years after surgery. During the follow-up, 5 patients lost contact, and a total of 53 people were followed up. The postoperative evaluation indexes mainly include WOMAC, $\mathrm{KSS}$, and VAS scoring etc. In addition, we introduced the FJS scoring in this mid-term study, which can more accurately reflect the post-TKA knee joint functions. The follow-up data collection mainly adopted the form of questionnaires. Patients and their families were required to fill in the relevant scoring questionnaires, and dedicated personnel were assigned to collect the data in conjunction with visualized quantitative scoring, professional knee joint physical examination and follow-up data collection.

The surgeries were performed by the same senior professional physician with a post-stable knee prosthesis system (PS Scorpio NRG PS, Stryker). Conventional midline anterior knee incision and medial parapatella approach were used to treat the femoral condyle, tibial plateau and patella sequentially, the peripatellar soft tissues and prepatellar fat pad were loosened, and the patella osteophytes were removed. Patella arthroplasty was performed on both groups, and no patella replacement was performed on either group during the surgeries. Patients were randomly divided into experimental group and control group according to the random number table prepared before the surgeries. The grouping information was completed with the assistance of nurses and stored in a specific envelope to be collated after unblinding. The experimental group underwent patella arthroplasty and patella denervation treatment, and the control group only underwent patella arthroplasty. Patella denervation adopted the current mainstream operating standards, the electric knife was set at $50 \mathrm{w}$, and the patella peripheral cauterization depth was about $5 \mathrm{~mm}$, and the patella track was checked after the placement of prosthesis. Drainage tubes were placed and patients can walk within 24 hours after surgery. Postoperative standard anticoagulation and multimodal analgesia treatments, and postoperative functional rehabilitation were carried out. Data analysis was performed using SPSS ver 21.0 (SPSS Inc., Chicago, IL, USA). The KSS score, WOMAC score, and FJS score were statistically analyzed by independent t-test, and the p-value was $<0.05$.

\section{Results}

A total of 58 patients were enrolled in this study. One case was lost to follow-up due to sudden cardiac death after surgery in the early follow-up, and 5 people lost contact during the mid-term follow-up. A total of 53 people were followed up to 3 and 5 years after surgery, with 11 cases of males, and 42 cases of females, average age 68.7 (57-80 years) years old. There was no statistically significant difference between the groups.

This study is a randomized controlled double-blind 
experiment, using self-comparison, it was determined that the age and weight of preoperative patients are not interfering factors, and there was no statistical difference in KSS, WOMAC, etc. before surgery (Table 1, 2). The average ROM of the experimental group after surgery was not statistically different in the 3-year and 5-year follow-up from the control group (Table 1). Similarly, we found that the differences in KSS and WOMAC scores between the control group and the experimental group followed up at 3 and 5 years after surgery were also not statistically significant (Table 2). The two groups had statistically significant differences in FJS scores.

Table 1. Statistics of ROM and VAS before surgery, in the 3-year and 5-year follow-up

\begin{tabular}{|c|c|c|c|}
\hline Results & Experimental Group & $\begin{array}{l}\text { Control Group } \\
\qquad \begin{array}{c}(\mathbf{n}=\mathbf{5 3}) \\
\mathbf{x} \pm \mathbf{s}\end{array}\end{array}$ & $\begin{array}{c}\mathbf{P} \\
(\mathbf{n}=\mathbf{5 3}) \\
\mathbf{x} \pm \mathbf{s}\end{array}$ \\
\hline \multicolumn{4}{|l|}{ ROM: } \\
\hline Before Surgery & $86.19 \pm 3.00$ & $85.91 \pm 3.51$ & 0.642 \\
\hline 3 Years after Surgery & $125.33 \pm 3.19$ & $124.32 \pm 3.92$ & 0.979 \\
\hline 5 Years after Surgery & $133.49 \pm 3.24$ & $135.47 \pm 3.12$ & 0.927 \\
\hline \multicolumn{4}{|l|}{ VAS } \\
\hline Before Surgery & $7.72 \pm 1.10$ & $8.00 \pm 0.87$ & 0.133 \\
\hline 3 Years after Surgery & $2.10 \pm 0.91$ & $2.05 \pm 0.85$ & 0.426 \\
\hline 5 Years after Surgery & $1.1 \pm 0.92$ & $1.02 \pm 0.87$ & 0.463 \\
\hline
\end{tabular}

$\mathrm{ROM}=$ Range of motion, VAS=Visual Analogue Scale

Table 2. Statistics of WOMAC and KSS scores and FJS before surgery, in the 3-year and 5-year follow-up

\begin{tabular}{|c|c|c|c|}
\hline Scoring & $\begin{array}{l}\text { Control Group } \\
\qquad \begin{array}{c}(\mathbf{n}=\mathbf{5 3}) \\
\mathbf{x} \pm \mathbf{s}\end{array}\end{array}$ & $\begin{array}{l}\text { Experimental Group } \\
\qquad \begin{array}{c}(\mathbf{n}=\mathbf{5 3}) \\
\mathbf{x} \pm \mathbf{s}\end{array}\end{array}$ & $\mathbf{P}$ \\
\hline WOMAC Score & $58.57 \pm 4.30$ & & \\
\hline Before Surgery & $16.61 \pm 4.28$ & $58.52 \pm 4.35$ & 0.948 \\
\hline 3 years after surgery & $10.05 \pm 4.88$ & $15.45 \pm 3.31$ & 0.268 \\
\hline 5 years after surgery & $28.23 \pm 2.09$ & $10.46 \pm 2.21$ & 0.592 \\
\hline \multicolumn{4}{|l|}{ KSS Score } \\
\hline \multicolumn{4}{|l|}{ Function score } \\
\hline Before Surgery & $35.27 \pm 2.37$ & $35.70 \pm 1.57$ & 0.184 \\
\hline 3 years after surgery & $82.72 \pm 2.27$ & $83.93 \pm 2.22$ & 0.751 \\
\hline 5 years after surgery & $92.95 \pm 2.71$ & $93.96 \pm 2.58$ & 1.010 \\
\hline \multicolumn{4}{|l|}{ FJS } \\
\hline Before Surgery & $30.80 \pm 1.85$ & $31.21 \pm 1.83$ & 0.759 \\
\hline 3 years after surgery & $88.25 \pm 4.59$ & $92.07 \pm 5.19$ & 0.041 \\
\hline 5 years after surgery & $89.00 \pm 5.24$ & $95.66 \pm 6.24$ & 0.049 \\
\hline DBP & $70.46 \pm 4.43$ & $9.96 \pm 5.63 *$ & $3 \pm 10.53 * \#$ \\
\hline
\end{tabular}

WOMAC $=$ Western Ontario and McMaster Universities.

\section{Discussion}

The early phase study found that the control group and the experimental group had no statistically significant differences in KSS score, WOMAC, VAS and other 
postoperative knee joint functions through statistical analysis of the follow-up data at 3 and 12 months after surgery. It is believed that patella denervation has no significant effects on the postoperative knee joint functional recovery in TKA without patella replacement. Due to the relatively short postoperative follow-up time in the initial study, further follow-up cannot be used to understand the effects of patella denervation on long-term postoperative knee joint functions. Therefore, this study extended the follow-up time to further study the effects of patella denervation surgery on knee joint functions after TKA. According to the analysis of follow-up data at 3 and 5 years after surgery, the control group and the experimental group have statistically significant differences in postoperative FJS scores in the mid-term follow-up after surgery. Patella denervation can positively improve postoperative knee joint functions significantly; the knee joint functions feel better after surgery. We believe that patella denervation during TKA has a positive effect on postoperative knee functional recovery, and is especially beneficial to long-term knee functional recovery.

Patella denervation was first proposed by Vega et al ${ }^{[9]}$ and used in clinical practice. Patella perimeter electrocautery is one of the safe and effective surgical procedures, and it has been widely used in China and abroad. The denervation of the patella is achieved by cauterizing the soft tissues around the patella at high temperature to block the patellar nerve pathway and destroy the patellar pain receptors, thereby reducing the occurrence of postoperative anterior knee pain and improving postoperative knee joint functions. Currently, most surgeons use $50 \mathrm{~W}$ power setting to perform circular cauterization around the patella with a depth of about $5 \mathrm{~mm}^{[14]}$. In theory, denervation around the patella is very effective in relieving anterior knee pain. High temperature cauterization can destroy the nerve plexus around the patella, and some scholars believe that it reduces the pain receptors in the soft tissues around the patella to achieve a fundamental reduction in the occurrence of anterior knee pain. The specific principle remains to be further studied.

There are many studies on the postoperative effects of patella denervation after TKA surgery, but the results are quite different, and even contradictory results are observed. The influence of patella denervation on postoperative functional recovery is still controversial. For example, Baliga $^{[15]}$ found that denervation did not improve the postoperative functions after total knee arthroplasty. The same results were observed in patients with TKA who underwent patella replacement. However, Gupta ${ }^{[16]}$ and Breugem $\mathrm{SJ}^{[17]}$ found that this technique can significantly improve the incidence of postoperative knee pain and postoperative joint function scores. Yim et al ${ }^{[18]}$ compared 100 TKA patients with 1-year follow-up and found that the experimental group and the control group had no statistically significant differences in KSS, ROM, and patella scores. Although they also included patients with bilateral knee replacement during the same period and used commonly used indicators such as KSS and WOMAC, but the shortcomings were that the follow-up time was relatively short, and the surgeries were carried out by different surgeons and different designs of prostheses were used. Factors such as system, surgical technique differences between surgeons, and prosthesis design can easily affect the research results. This study was carried out by the same surgeon using the same prosthesis system. Through self-comparison, confounding factors were found to have little interference on the results and higher reliability. Related researches in the literature also support that prosthesis design and surgical technique also have important influences on postoperative functional recovery ${ }^{[19,20]}$.

Fan et $a l^{[2]}$ conducted a meta-analysis which included six randomized controlled clinical studies. It was concluded that denervation of the patella during TKA without patella replacement is beneficial to the recovery of knee joint functions after surgery, but has no significant effects on the incidence of anterior knee pain. A meta-analysis conducted by Xie et $a l^{[21]}$ found that regarding morbidity, VAS, patella score, and range of motion, patella denervation can significantly improve clinical outcomes in the first 12 months after surgery. However, in the follow-up of more than 12 months, no difference in scores was found, which indicates that the initial advantages of patellar denervation seem to have faded. One of the trials included assessments at 3 , 12, and 24 months after surgery. The study found that compared with the non-denervation treatment at 3 months, the incidence of anterior knee pain in the patella denervation operation group was significantly lower, but not at 12 or 24 months, indicating the time of continuous follow-up may have a great influence on the relationship between patella denervation and treatment of anterior knee pain. In this study, through longer follow-up and data collection, the denervated patella group is more advantageous in terms of FJ score, and the knee joint has better feeling and functions in the long 
term. It has a great impact on the correlation with the incidence of AKP.

Currently, there is no consensus and unified technical standard for the patella denervation surgery. The operation of the surgeon such as the power of the electrosurgical knife and the degree of cauterization may affect the efficacy of the denervation surgery. The accurate grasp of the operation depth and range depends on the habits of the surgeons, and these confounding factors may reduce the comparability between the individuals and reduce the reliability of the study. In order to reduce the interference of these confounding factors on the results, our research was performed by the same experienced surgeon with the same fixed-platform bone-cement type post-stabilizing knee prosthesis. Interference factors due to surgical operation and false design, etc. are eliminated as much as possible, and the reliability of the study is increased by further extending the follow-up time. Meanwhile, random double-blind grouping is used to avoid selection bias.

An RCTS follow-up analysis of 2 years by Ramnadh et $a l^{[22]}$ found that the effects of patellar denervation was statistically significant in the patella score and postoperative VAS score 3 months after surgery, but there was no statistical difference in the long-term. Gupta ${ }^{[23]}$ also had a study that came to a similar conclusion to ours. After two years of follow-up, it was found that denervation of the patella was not beneficial to the postoperative efficacy. However their study was not a randomized controlled study but a retrospective matched study. Confounding factors such as selection bias may affect the conclusions of the study, which may reduce the reliability of the study. Wojtys et al ${ }^{[11]}$ also reported that substance $P$ was found around the nerve fibers in the soft tissues of the patella, which provided a theoretical basis for denervation of the patella.

Goicoechea $\mathrm{N}^{[24]}$ found that denervation of the patella had no significant effect on reducing anterior knee pain and improving postoperative knee joint functions. Therefore, denervation of the patella during TKA can be omitted, and this procedure is not recommended. A meta-analysis conducted by Peng $\mathrm{L}^{[25]}$ showed that TKA patella denervation can improve knee mobility and reduce the incidence of postoperative anterior knee pain. Therefore, it is recommended that this operation be routinely performed during TKA. Similar to the results of this study, the denervation of the patella has a positive effect on the long-term recovery of knee joint functions. Gao Qiang et $a l^{[26]}$ found that an important preventive measure for anterior knee pain after TKA was achieved by effective intraoperative patella denervation, which was similar to the results of this study.

This clinical randomized controlled research uses a follow-up time of up to 5 years, and the information is complete. The follow-up time was further increased on the basis of the initial follow-up, and the reliability is higher. Meanwhile, we innovatively introduce the FJS function score, which is more realistic and objective. Evaluation of postoperative functions is of great significance for clinical guidance. However, there are some shortcomings. For example, the sample size is small, and there is no uniform standard for patella denervation, and its efficacy is affected by the operation of the surgeon. And due to the subjective perception of pain, and the individual's pain threshold varies from person to person, data collection is mainly done through quantitative forms and visual scoring, and collection of medical history. These individual-related differences cannot be ruled out in the study and may have an impact on the validity of the results, but as the sample size is gradually increased and the follow-up time is prolonged, its impact can be minimized, and the reliability of randomized controlled studies can be further improved to better guide clinical practice.

\section{Conclusion}

We believe that TKA patella denervation has positive effects on knee joint functions in the mid- and long-term, and the postoperative satisfaction is better, and the FJS score is higher. This surgical operation is recommended in clinical work to facilitate postoperative functional recovery.

\section{References}

[1] Duan G, Liu C, Lin W, Shao J, Fu K, Niu Y, Wang F. Different Factors Conduct Anterior Knee Pain Following Primary Total Knee Arthroplasty: A Systematic Review and Meta-Analysis. J Arthroplasty. 2018 Jun; 33(6):1962-1971.e3.

[2] Fan L, Ge Z, Zhang C, Li J, Yu Z, Dang X, Wang K. Circumferential electrocautery of the patella in primary total knee replacement without patellar replacement: a meta-analysis and systematic review. Sci Rep. 2015 Mar 24; 5:9393.

[3] Metsna V, Vorobjov S, Lepik K, Märtson A. Anterior knee pain following total knee replacement correlates 
with the OARSI score of the cartilage of the patella. Acta Orthop. 2014 Aug;85(4):427-32. [DOI: 10.3109/17453674.2014.931198 EPUB 2014 Jun 23 PMID: 24954482 PMCID: PMC4105776].

[4] Ritter MA, Campbell ED. Effect of range of motion on the success of a total knee arthroplasty. J Arthroplasty. 1987; 2(2):95-7.

[5] Wood DJ, Smith AJ, Collopy D, White B, Brankov B, Bulsara MK. Patellar resurfacing in total knee arthroplasty: a prospective, randomized trial. J Bone Joint Surg Am. 2002 Feb; 84(2):187-93.

[6] Pilling RW, Moulder E, Allgar V, Messner J, Sun Z, Mohsen A. Patellar resurfacing in primary total knee replacement: a meta-analysis. J Bone Joint Surg Am. 2012 Dec 19; 94(24):2270-8.

[7] Pavlou G, Meyer C, Leonidou A, As-Sultany M, West $\mathrm{R}$, Tsiridis E. Patellar resurfacing in total knee arthroplasty: does design matter? A meta-analysis of 7075 cases. J Bone Joint Surg Am. 2011 Jul 20; 93(14):1301-9.

[8] Lygre SH, Espehaug B, Havelin LI, Vollset SE, Furnes O. Does patella resurfacing really matter? Pain and function in 972 patients after primary total knee arthroplasty. J Acta Orthop. 2010 Feb; 81(1):99-107.

[9] Vega J, Golanó P, Pérez-Carro L. Electrosurgical arthroscopic patellar denervation. J Arthroscopy. 2006 Sep; 22(9):1028.e1-3.

[10] van Jonbergen HP, Barnaart AF, Verheyen CC. A dutch survey on circumpatellar electrocautery in total knee arthroplasty. J Open Orthop . 2010 Jun 22; 4:201-3.

[11] Wojtys EM, Beaman DN, Glover RA, Janda D. Innervation of the human knee joint by substance-P fibers. J Arthroscopy. 1990; 6(4):254-63.

[12] Spencer BA, Cherian JJ, Margetas G, Chughtai M, Harwin SF, Elmallah RK, Mont MA. Patellar resurfacing versus circumferential denervation of the patella in total knee arthroplasty. J Orthopedics. 2016 Sep 1; 39(5):e1019-23.

[13] Ahay MA, Ertiirk C, Ahay N, et a1. Patellar denervation in total knee arthroplasty without patellar resuffaeing: A prospective, ran-domized controlled study. J Orthop Traumatol Surg Res, 2012, 98(4): 421-425.

[14] Banon RS, Ostrowski ML, Anderson TD, et al. Intraosseous in-nervation of the human patella : A histologic study. J Am J Med, 2007, 35(2): 307-311.
[15] Baliga S, McNair CJ, Barnett KJ, MacLeod J, Humphry RW, Finlayson D. Does circumpatellar electrocautery improve the outcome after total knee replacement? A prospective, randomised, blinded controlled trial. J Bone Joint Surg Br. 2012 Sep; 94(9):1228-33.

[16] Gupta S, Augustine A, Horey L, Meek RM, Hullin MG, Mohammed A. Electrocautery of the patellar rim in primary total knee replacement: beneficial or unnecessary? J Bone Joint. Surg Br. 2010 Sep; 92(9):1259-61.

[17] Breugem SJ, Sierevelt IN, Schafroth MU, Blankevoort L, Schaap GR, van Dijk CN. Less anterior knee pain with a mobile-bearing prosthesis compared with a fixed-bearing prosthesis. J Clin Orthop Relat Res. 2008 Aug; 466(8):1959-65.

[18] Yim SJ, Jang MS, Kim WJ, Lee SH, Kang HK. The Effect of electrocautery around the patellar rim in patellar non-resurfacing total knee arthroplasty. J Knee Surg Relat Res. 2012 Jun; 24(2):104-7.

[19] Maculé F, Sastre S, Lasurt S, Sala P, Segur JM, Mallofré C. Hoffa's fat pad resection in total knee arthroplasty. J Acta Orthop Belg. 2005 Dec; 71(6):714-7.

[20] Pulavarti RS, Raut VV, McLauchlan GJ. Patella denervation in primary total knee arthroplasty - a randomized controlled trial with 2 years of follow-up. J Arthroplasty. 2014 May; 29(5):977-81.

[21] Xie X, Pei F, Huang Z, Tan Z, Yang Z, Kang P. Does patellar denervation reduce post-operative anterior knee pain after total knee arthroplasty? J Knee Surg Sports Traumatol Arthrosc. 2015 Jun; 23(6):1808-15.

[22] Pulavarti RS, Raut VV, McLauchlan GJ. Patella denervation in primary total knee arthroplasty - a randomized controlled trial with 2 years of follow-up. $\mathrm{J}$ Arthroplasty. 2014 May; 29(5):977-81.

[23] Gupta S, Augustine A, Horey L, Meek RM, Hullin MG, Mohammed A. Electrocautery of the patellar rim in primary total knee replacement: beneficial or unnecessary? J Bone Joint Surg Br. 2010 Sep; 92(9):1259-61.

[24] Goicoechea N, Hinarejos P, Torres-Claramunt R, Leal-Blanquet J, Sánchez-Soler J, Monllau JC. Patellar denervation does not reduce post-operative anterior knee pain after primary total knee arthroplasty with patellar resurfacing. Knee Surg Sports Traumatol Arthrosc, 2020 Aug 5. 
[25] Peng L, Luo Y, Liu J, Li Z. The efficacy of patellar denervation with electrocautery after total knee replacement: A meta-analysis of randomized controlled trials. Int J Surg, 2020 Jun; 78:126-137.
[26] Gao Q, Yao YF. Causes and treatment of anterior knee pain after total knee arthroplasty. J Chinese Journal of Bone and Joint Injury, 2019, 34(12):1340-1343. 